\title{
Multi-Fractality in Foreign Currency Markets
}

\author{
Marco Corazza \\ University Ca' Foscari of Venice, Italy \\ A. G. Malliaris \\ Loyola University Chicago, U.S.A.
}

Several empirical studies have shown the inadequacy of the standard Brownian motion $(\mathrm{sBm})$ as a model of asset returns. To correct for this evidence some authors have conjectured that asset returns may be independently and identically Pareto-Lévy stable (PLs) distributed, whereas others have asserted that asset returns may be identically - but not independently - fractional Brownian motion ( $\mathrm{fBm}$ ) distributed with Hurst exponents, in both cases, that differ from 0.5. In this article we empirically explore such non-standard assumptions for both spot and (nearby) futures returns for five foreign currencies: the British Pound, the Canadian Dollar, the German Mark, the Swiss Franc, and the Japanese Yen.

Keywords: exponent of Hurst, fractional Brownian motion, multi-fractal market hypothesis, Pareto-Lévy stable process, R/S analysis.

\section{Introduction}

The standard hypothesis concerning the behavior of asset returns in financial markets claims that they are independently and identically lognormally distributed $\left(\ln \left[P(t+d t)-\ln [P(t)] \sim N\left(\mu d t, \sigma^{2} d t\right)\right)\right.$. The

\footnotetext{
*The first draft of this article was written when Marco Corazza was a Visiting Research Associate in the Department of Economics at Loyola University Chicago. Earlier versions of this article were presented in the Asset and Liability Management Conference, The University of British Columbia, Vancouver, B.C., Canada, August 14-16, 1998 and at the Eighth Annual Conference of the Multinational Finance Society in Verona, Italy, June 2327, 2001. The authors are grateful to two anonymous referees for several helpful suggestions, to Carolyn Tang and Robert Gyorgy for editorial assistance and to Peter Theodossiou for his generous encouragement.
}

(Multinational Finance Journal, 2002, vol. 6, no. 2, pp. 65-98)

(CMultinational Finance Society, a nonprofit corporation. All rights reserved.

DOI: $10.17578 / 6-2-1$ 
corresponding underlying stochastic process is characterized by a quantity, called the Hurst exponent $H$, which is related to some fractal aspects of the process itself. ${ }^{1}$ In particular, for a standard Brownian motion (sBm) the Hurst exponent is $H=0.5$.

Several empirical studies have supported the independent and identical lognormal behavior of asset returns, but others have shown its inadequacy as a model of asset returns. This inadequacy is often caused by the existence of many outliers, nonstationarity in the variance level, presence of asymmetry, and short and long-term dependence. Authors such as Lo and MacKinlay (1988), Lo (1991), Peters (1991, 1994), Evertsz (1995a, 1995b), Evertsz and Berkner (1995), Corazza (1996), Campbell, Lo and MacKinlay (1997) and Corazza, Malliaris and Nardelli (1997) provide statistical evidence that asset prices do not follow random walks.

To account for this discrepancy, some authors have conjectured that financial returns may be independently and identically Pareto-Lévy stable (PLs) distributed, whereas others have conjectured that asset returns may be identically, but not independently, fractional Brownian motion (fBm) distributed. ${ }^{2,3}$ Both of these conjectures are characterized by exponents of Hurst such that $H \neq 0.5$.

In this article we consider such non-standard hypotheses about returns for both spot and (nearby) futures for five foreign currency markets: the British Pound, the Canadian Dollar, the German Mark, the Swiss Franc and the Japanese Yen. We assume the Hurst exponent $H$ belongs to a suitable neighborhood of 0.5 , that is, we (indirectly) assume that the stochastic process generating exchange rate returns can be either a PLs or a fBm motion. This assumption provides a more flexible theoretical framework to examine if the so-called Fractal Market

1. There is extensive literature on fractality from a mathematical point of view, such as Mandelbrot and Van Ness (1968) and Falconer (1990). Applications of fractality in finance are presented in Evertsz (1995a, 1995b), Evertsz and Berkner (1995), and Corazza, Malliaris and Nardelli (1997).

2. See Mittnik and Rachev (1993) and Campbell, Lo and MacKinlay (1997).

3. Representative references include Lo (1991), Peters (1991, 1994), Corazza (1996), Evertsz (1995a, 1995b), Evertsz and Berkner (1995), Belkacem, Levy Vehel, and Walter (1996), Ostasiewicz (1996), Campbell, Lo and MacKinlay (1997) and Corazza, Malliaris and Nardelli (1997). 
Hypothesis (FMH), as proposed in Peters $(1991,1994)$, is a reasonable generalization of the standard Efficient Market Hypothesis (EMH), initially elaborated in Fama (1970). Of course, when $H=0.5$ the FMH coincides with the EMH. Furthermore, we also assume that the Hurst exponent is a function of time, $H=H(t)$, allowing the foreign currency markets structures to vary over time. The introduction of this dynamic dimension permits the generalization of the FMH into the MultiFractal Market Hypothesis (MFMH).

Briefly, the MFMH provides a theoretical framework to account for changes from "regular" to "irregular" phases of the capital markets and vice versa. In general, in such markets traders have investment horizons with similar or different lengths. If the matching between the asset demand and supply is relatively equal, then both the liquidity and regularity of the markets are ensured, otherwise the opposite holds. ${ }^{4,5}$ Of course, when $H=0.5$, for all suitable $t$, then the MFMH coincides with the EMH.

The remainder of the article is organized as follows: in section 2 we give a brief review of the literature; in sections 3 and 4 we present some theoretical and empirical aspects that are essential to our analysis; section 5 describes the data and section 6 reports the results of the multifractal analysis. In section 7 we offer an economic interpretation of our results, and finally, in section 8 , we summarize our concluding remarks.

\section{Review of the Literature}

Market efficiency has been the most celebrated theory of financial markets during the past three decades. In its simplest formulation this theory claims that changes in asset prices reflect fully and instantaneously the release of all new relevant information. Furthermore, because such a flow of information cannot be anticipated between the current trading period and the next one, asset price changes, in efficient markets, are serially independent. In other words, the release of

\footnotetext{
4. Notice that the peculiarities of such a matching depends on the stochastic process generating the asset returns.

5. These concepts are discussed in detail in Pancham (1994), Corazza (1996), Belkacem, Vehel, and Walter (1996) and also in section 6 of this article.
} 
unanticipated information moves asset prices randomly. The textbook by Campbell, Lo and MacKinlay (1997, section 8) explains various versions of the random walk hypothesis.

The efficient market theory, from its earliest formulation by Samuelson (1965) and Fama (1970), has been refined in several directions. Analytically, the concept of information has been rigorously defined. Statistically, the notion of random walk has been generalized to Itô processes. Moreover, the efficient market hypothesis has been extensively tested. Fama (1991) traces the evolution of the market efficiency theory during its first two decades and skillfully cites numerous studies that offer empirical support as well as empirical rejection of the EMH.

In this article we conduct an empirical investigation of the return behavior of five foreign currencies in order to detect possible discrepancies between the actual behavior of such currencies and the classical random walk. Note that we do not claim that foreign currency markets are inefficient nor do we assert that the EMH does not hold. We acknowledge that market efficiency is currently the central theory of financial economics, at least until a new theory is proposed as a better explanatory paradigm of asset prices behavior. We merely wish to emphasize the need for revising the $\mathrm{EMH}$ and provide empirical evidence to this end.

The existing literature proposes several approaches for verifying whether a foreign exchange market is more or less efficient. In the remainder of this section we briefly review some of most significant findings.

From an econometric standpoint, Cornell (1977), Frankel (1980), Chiang and Jiang (1995), and Zhou (1996) examine whether the current spot, the forward rate or the futures price can be used as an unbiased predictor of the spot rate itself at some future date. From the same point of view, it is possible to use the recent time series tools of cointegration, ARCH and GARCH techniques to detect possible market inefficiencies. Kao and Ma (1992), Leachman and El Shazly (1992), Chan, Gup and Pan (1992) and Alexakis and Apergis (1996) utilize such methodologies.

A more operative approach consists of devising certain trading rules concerning these markets and determining their profitability, as in Taylor (1992), Levich and Thomas (1993), and Kho (1992).

A third class of techniques looks for deterministic nonlinear and chaotic dynamics in foreign currency market data. Hsieh $(1988,1992)$, and 
Bleaney and Mizen (1996) follow these methodologies.

Finally, a recent "inter disciplinary" approach is the fractal one which is linked to both stochastic and deterministic aspects of the underlying process generating the price changes. The tools of fractal analysis are employed by Liu and Hsueh (1993), Fang, Lai and Lai (1994), Evertsz (1995a, 1995b), Evertsz and Berkner (1995), Van de Gucht, Dekimpe and Kwok (1996), Corazza, Malliaris and Nardelli (1997) and in this article. A detailed presentation of these techniques is given in Shubik (1997).

\section{Theoretical Aspects}

The current literature proposes different stochastic processes to describe the behavior of financial returns. The most common approaches are the fractional Brownian motion (fBm), and some of the Pareto-Levy stable (PLs) distribution sub-families. In general, these stochastic processes can be characterized by the same Hurst exponent, $H \neq 0.5$, as explained in Taqqu (1986), Evertsz (1995a, 1995b), and Evertsz and Berkner (1995). In fact, if such stochastic processes are independently and identically distributed with exponentially decaying power-law tails, as for example the PLs, then $H \in(0.5,1)$, whereas if they are identically, but not independently distributed, as for example the fBm, then $H \in(0,1){ }^{6}$

In order to conduct our analysis and consequently to test the MultiFractal Market Hypothesis (MFMH), we need a set of mathematical and statistical tools to formally define and estimate the long-term dependence of asset returns and to determine the value of the Hurst exponent. In particular, in this section we first define the fBm and PLs motions and present some of their properties. Second, we describe some tests for detecting long-term memory in time series and we introduce some algorithms for estimating the Hurst exponent, $H$.

\section{A. Fractional and MultiFractional Brownian Motion}

The $\mathrm{fBm}$ is a term coined by Mandelbrot and Van Ness (1968) to

6. Notice that the interval $(0.5,1)$ is obtained as the intersection of the ones characterizing each of the different PLs distribution sub-families. Taqqu (1986) includes in these subfamilies, the symmetric, the fractional and the log-fractional one, among others. 
describe an almost everywhere continuous Gaussian stochastic process of index $H \in(0,1),\left\{B_{H}(t), t \geq 0\right\}$, defined by a Riemann-Liouville stochastic integral, such that $B_{H}(0)=0$ with probability 1 , and that $B_{H}\left(t_{2}\right)$ $-B_{H}\left(t_{1}\right) \sim N\left(0, \sigma^{2 H}\left(t_{2}-t_{1}\right)^{2 H}\right)$, with $0 \leq t_{1}<t_{2}<+\infty$ and $\sigma>0$. In particular, if $H \neq 0.5$ then the increments are stationary but not independent, and they show a long-term memory depending on both $H$ and $t_{2}-t_{1}$. If $H \in(0,0.5)$, there is a negative dependence between the increments. In this case the stochastic process has an anti-persistent behavior. If $H \in(0.5,1)$, there is a positive dependence between the increments and in this case the process has a persistent behavior. The case $H=0.5$ is the sBm that has independent increments. Moreover, this stochastic process is statistically self-similar, that is $\left\{B_{H}(t), t \geq 0\right\}$ and $\left\{a^{-H} B_{H}(a t), t \geq 0\right\}$, with $a>0$, have the same distribution law. Further details for the fBm can be found in Falconer (1990), Evertsz (1995a, 1995b), Evertsz and Berkner (1995) and Corazza, Malliaris and Nardelli (1997).

In 1995, Peltier and Levy (1995) proposed an extension of the fBm by substituting the constant over time Hurst exponent, $H$, with a suitable time dependent function, $H(t)$. Unlike the $\mathrm{fBm}$, this new stochastic process, called multifBm ( $\mathrm{mfBm})$, allows us to formally model the irregularities of the process trajectory. As such, this stochastic process can be fruitfully utilized to describe non-stationarity in financial asset price variations. ${ }^{7}$

\section{B. Pareto-Lévy Stable Stochastic Process}

The PLs motion, originally introduced by Lévy (1925) as a generalization of the sBm, is a stochastic process, $\left\{L_{\alpha}(t), t \geq 0\right\}$, characterized by a distribution, $S_{\alpha, \beta}(\mu, \sigma)$, depending on four parameters: the so-called characteristic exponent $\alpha \in(0,2]$, the skewness parameter $\beta \in[-1,1]$, the location parameter $\mu \in(-\infty,+\infty)$, and the scale coefficient $\sigma \in[0$, $+\infty) .{ }^{8}$ This stochastic process is such that $L_{\alpha}(0)=0$ almost-surely, and its increments $L_{\alpha}\left(t_{2}\right)-L_{\alpha}\left(t_{1}\right)$, with $0 \leq t_{1}<t_{2}<+\infty$, whose distribution is $S_{\alpha, \beta}\left(0,\left(t_{2}-t_{1}\right)^{1 / \alpha}\right)$, are independent and stationary. In particular, if $\alpha$

7. For details, see Cheung and Lai (1993), Corazza (1996) and Belkacem, Levy and Walter (1996).

8. If $\alpha \in(0,1)$ the distribution does not have a finite mean or a finite variance. If $\alpha \in$ $[1,2)$ the distribution has only a finite mean and if $\alpha=2$, the distribution has both finite mean and finite variance. 
$\in(0,2)$ then the tails of such a process decay slower than the tails of an $\mathrm{fBm}$ process, and if $\alpha=2$ it is possible to prove that $\left\{2^{-1 / 2} L_{2}(t), t \geq 0\right\}$ $\equiv\left\{B_{0.5}(t), t \geq 0\right\}$, which is the $\mathrm{sBm}$. Moreover, if the distribution $S_{\alpha, \beta}(\mu$, $\sigma)$ is symmetric, that is if $\beta=0$, then the corresponding PLs process is statistically self-similar. ${ }^{9}$ Taking $\left\{L_{\alpha}(t), t \geq 0\right\}$ and $\left\{a^{-1 / \alpha} L_{\alpha}(a t), t \geq 0\right\}$, with $a>0$, results in the same distribution law. In such a case it is possible to prove that the Hurst exponent equals $H=1 / \alpha .^{10}$

\section{Empirical Aspects}

Although a large empirical literature exists confirming the presence of long-run memory or long-range dependence in asset prices, there are no universally accepted quantitative methodologies that make it possible to detect such long-term dependence in (finite) time series as argued by Taqqu, Teverovsky and Willinger (1995). Moreover, some of the methodologies used show considerable limitations. Thus, in order to overcome the shortcomings of each methodology, we follow two different inferential approaches and compare the corresponding results. The methodologies employed are the classical modified range over standard deviation statistic, $R / S$, and the periodogram approach. ${ }^{11}$

\section{A. Tests for Long-Term Dependence}

\section{i. The Modified R/S Test}

Lo (1991) proposes a modification of a test based on the classical range over standard deviation statistic, $R / S$. To test for no long-term dependence in financial time series consider:

$$
Q_{r}(q)=\frac{R_{T} / S_{T}(q)}{\sqrt{T}}
$$

9. From a financial standpoint it is not restrictive to assume that $\beta=0$. In fact, most of the skewness parameters estimated from asset returns time series, though different from 0 , are quite close to it.

10. See Taqqu (1986) and Corazza, Malliaris and Nardelli (1997).

11. We are grateful to an anonymous referee for suggesting that we use both methodologies. 
where $T$ is the time series size, $q$ is the possible short-term dependence (integer) length, $R_{T}(q)$ is the sample range of partial sums of deviations of the time series from its sample mean, and $S_{T}(q)$ is the modified standard deviation of the time series including the autocovariances weighted up to lag $q$. This new methodology is described in detail in both Lo (1991) and Campbell, Lo and MacKinlay (1997). Precisely, this statistic is able to test the null hypothesis of no long-term dependence. ${ }^{12}$ In particular, unlike the corresponding statistic based on the classical $R / S$, it is robust to short-term memory, conditional heteroscedasticity, and non-normal innovation. Furthermore, it also has well-defined distributional properties as described in Lo (1991) and Campbell, Lo and MacKinlay (1997), although the related (asymptotic) distribution is neither standard, nor easily tractable.

Of course, this statistic is crucially influenced by the statistical structure of short-term dependence. In order to accommodate this aspect, we apply two different approaches. In the first approach we specify in a nonparametric way the short-term memory structure determining the optimal value of $q$ by the use of the Andrews' (1991) data-dependent rule $q^{*}=\left[(3 T / 2)^{1 / 3}\left[\rho /\left(1-\rho^{2}\right)\right]^{2 / 3}\right]$, where the operator [.] denotes the greatest integer less than or equal to the argument, and $\rho$ is the sample first-order autocorrelation coefficient. In the second approach, we take into account the remarks of Lo (1991) and Jacobsen (1996) stating that, in general, there is little guidance in determining the optimal value of $q$. In this article, we follow the Jacobsen's (1996) procedure, and perform the test in two steps. First, we impose some specific models for the short-term dependence structure, namely an $\mathrm{AR}(1)$ one and a MA(1) one. ${ }^{13}$ Second, we apply the statistic $Q_{T}\left(q^{*}\right)$, with $q^{*}=0$, to the time series of the corresponding residuals.

Finally, by using the fractiles of the distribution of $Q_{T}(q)$ as in Lo

12. Notice that a rejection of such a null hypothesis does not necessarily imply that long-range dependence is present but, merely, that the underlying stochastic process does not simultaneously satisfy all the conditions stated by Lo (1991). However, such conditions are satisfied by many of the recently proposed stochastic processes for long-term dependence.

13. Notice that such an arbitrary way of choosing an AR(1) model and a MA(1) one is not particularly restrictive because, in general, such models are standard for handling short-term memory in financial returns time series. 
(1991), it is possible to determine critical values for different significance levels in this two-sided test. At 10, 5 and 1 percent they are 1.747, 1.862, and 2.098, respectively.

\section{ii. The Periodogram-based Test}

Lobato and Savin (1998) employ a suitable approximation to the Lagrange multiplier test in order to develop the no long-term dependence in the following time series statistic that is based on a periodogram and is given as such:

$$
L M_{T}(m)=m\left(\frac{\sum_{j=1}^{m} v_{j} I\left(\lambda_{j}\right)}{\sum_{j=1}^{m} I\left(\lambda_{j}\right)}\right)
$$

where $m$ is an (integer) bandwidth,

$$
v_{j}=\ln (j)-\left[\sum_{j=1}^{m} \ln (j)\right] / m,
$$

and

$$
I\left(\lambda_{j}\right)=\left[\left|\sum_{t=1}^{T} x_{t} \exp \left(i t \lambda_{j}\right)\right|\right] /(2 \pi T),
$$

is the periodogram computed at frequency $\lambda_{j}=(2 \pi j) / T$, in which $x_{t}$, with $t=1, \ldots, T$, is the time series, and $i=\sqrt{-1}$. More specifically, this statistic tests the null hypothesis $H_{0}: H=0.5$ rather than the alternative one $\mathrm{H}_{\mathrm{A}}: H \neq 0.5$. Moreover, this test is characterized by a well-known and quite tractable (asymptotic) distribution which is the $\chi_{1}^{2}$.

Of course, in this statistic, the bandwidth $m$ plays a crucial role. In order to determine its optimal value, we need to verify that certain proper assumptions hold ( $\operatorname{cuch}$ as the Gaussianity of $x_{t}$, with $t=1, \ldots, T$ ). We use the iterative algorithm presented in Delgado and Robinson (1996) to estimate $m$. We could also apply the widely used "rule of 
thumb" that sets $m=\sqrt{T}$.

Finally, by using the fractiles of the $\chi_{1}^{2}$ distribution, it is possible to determine the critical values for different significance levels for this twosided test.

\section{B. Procedure for Estimating the Hurst Exponent}

\section{i. The Modified R/S Estimation Procedure}

The Hurst exponent is linked to the modified $R / S$ statistic by $\lim _{\mathrm{t} \rightarrow+\infty}$ $E\left[R_{T} / S_{T}(q)\right] /\left(a T^{H}\right)=1$, with $a>0$. With this link it is possible to obtain the following approximate relationship: $\ln \left\{E\left[R_{T} / S_{T}(q)\right]\right\} \cong \ln (a)+H \ln (t)$. In order to estimate the value of the Hurst exponent, $H$, we have modified and improved the standard techniques described in Peters (1991, 1994), Corazza (1996) and Corazza, Malliaris and Nardelli (1997).

To do so, we first determine a series of estimates of the Hurst exponent $\left\{H_{j}, j=1, \ldots, T^{*}<T\right\}$ by fitting an ordinary least square regression between $\left.\left\{\ln \left[R_{T, l} / S_{T, l}(q)\right]\right\}, l=1, \ldots, j\right\}$ and $\{\ln (l), l=1, \ldots, j\}$, for every $j=2, \ldots, T^{*}$, where $R_{T, l}$ and $S_{T, l}(q)$ are quantities related to $R_{T}$ and $S_{T}(q)$ respectively. Then, we choose the optimal estimate in this series. Figures $1 \mathrm{~A}$ and $1 \mathrm{~B}$ illustrate the corresponding results for some of the analyzed time series by plotting $H_{j}$ versus $j$, with $j=2, \ldots, T^{*}$. In particular, this estimation procedure is robust, although possibly subject to bias, when the data generating process follows a highly non-normal distribution as argued by Lo (1991), Cheung and Lai (1993), Robinson (1994b), and Campbell, Lo and MacKinlay (1997). It is possible to prove its almost-sure convergence for stochastic processes with infinite variance. Consider for example the PLs distribution with $\alpha \in(0,2)$. Furthermore, Robinson (1994b) argues that the $R / S$ estimation procedure is suboptimal when the data generating process follows a Gaussian distribution because such a procedure does not depend on second moments.

Overall, the $R / S$-based estimation procedure described in this section offers the possibility to estimate the Hurst exponent without complete information, and without strong a priori assumptions on the distributional properties of the considered stochastic process. ${ }^{14}$

14. See Pancham (1994), Peltier and Levy (1994) and Taqqu, Teverovsky and Willinger (1995) for a different methodology. 


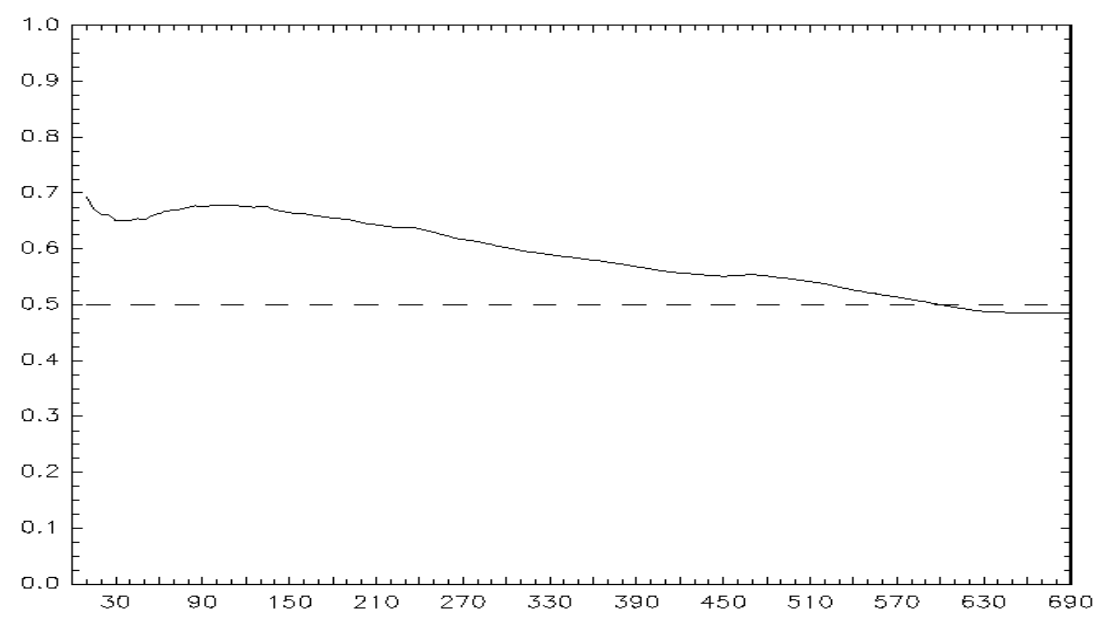

FIGURE 1A $-H$ versus $j$ for Canadian Dollar S. (08/76-01/82): The AR(1)case $\left(T^{*}=690\right)$.

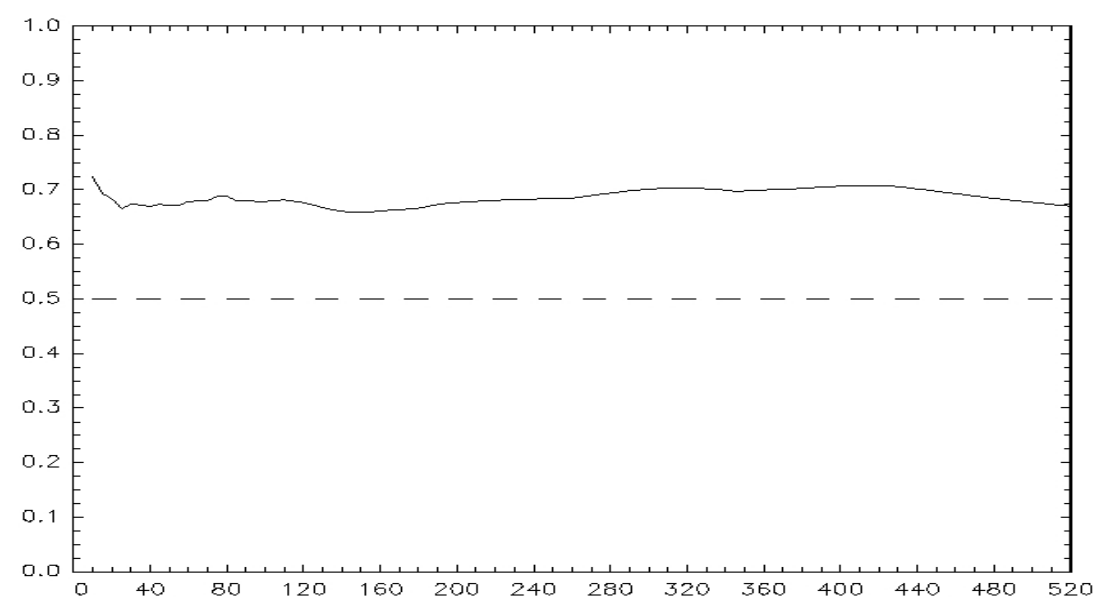

FIGURE 1B $-H$ versus $j$ for British Pound F. (06/72-07/76): The $q=\#$ case $\left(T^{*}=520\right)$

\section{ii. The Periodogram-based Estimation Procedure}

From a spectral density point of view, the Hurst exponent is linked to the discretely averaged periodogram: 


$$
F(\lambda)=2 \pi\left[\sum_{j=1}^{\lfloor\lambda T / 2 \pi\rfloor} I\left(\lambda_{j}\right)\right] / T .
$$

Starting from this relationship, Robinson (1994a) proposed the following closed form semi-parametric estimator for $H$ :

$$
H(m, r)=1-\frac{1}{2 \ln (r)} \ln \left[\frac{F\left(r \lambda_{m}\right)}{F\left(\lambda_{m}\right)}\right],
$$

where $m$ is the bandwidth introduced earlier and $r \in(0,1)$ is a suitable user-chosen variable. In particular, under the hypothesis that the datagenerating process follows a Gaussian distribution, it is possible to prove that this estimator is consistent and that it has well-defined (asymptotic) distributional properties both normal and non-normal, depending on the estimated value of $H(m, r){ }^{15,16}$

Of course $r$, plays a crucial role in this estimator. In particular, if some proper assumptions hold, among them the restrictions that $H \in$ $(0.5,0.75)$, then it is possible to determine its optimal value as discussed in Lobato and Robinson (1996). Thus, since both $m$ and $r$ depend on $H(m, r)$, in order to optimally estimate the Hurst exponent, we must determine a suitable series of converging estimates of $H,\left\{H_{j}\left(m_{j}, r_{j}\right), j\right.$ $=1, \ldots, J\}$. This can be done using the iterative algorithm proposed in Delgado and Robinson (1996). Figure 1C illustrates the corresponding results for one of the analyzed time series by plotting $H_{j}\left(m_{j}, r_{j}\right)$ versus $j$, with $j=1, \ldots, J$.

\section{Data Set and Descriptive Statistics}

The data we analyze are the time series of the daily returns using closing prices of exchange rates expressed in US Dollars, that is, $100\{\ln [P(t$ $+1)]-\ln [P(t)]\}$. We use data from June 1972 to September 1994, for the following five spot and (nearby) futures foreign currency markets:

15. See Robinson (1994a).

16. See Lobato and Robinson (1996). 


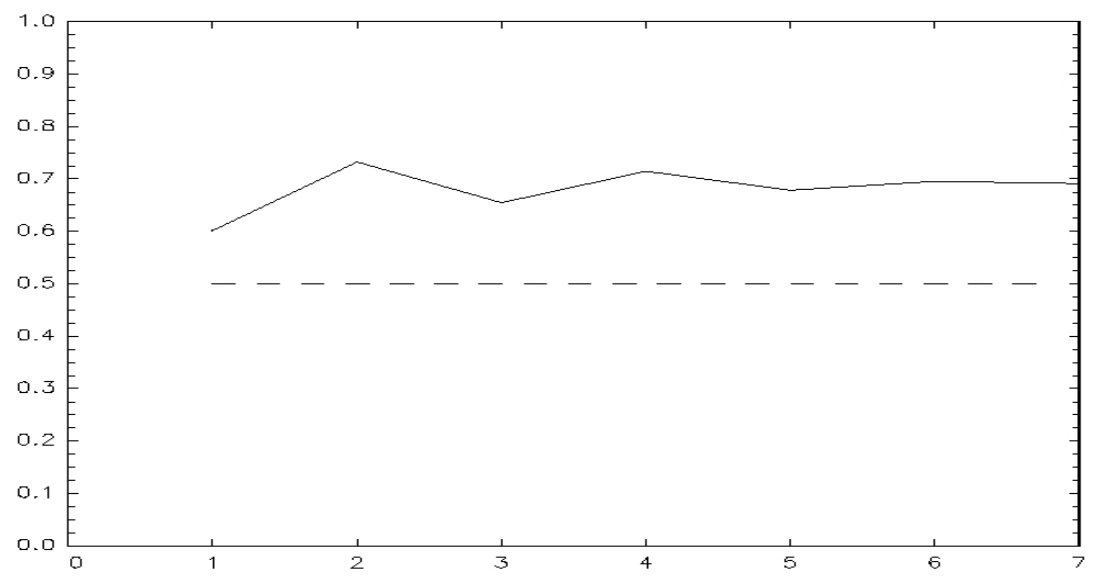

FIGURE 1C-H versus $j$ for German Mark F. (06/72-07/76): The Periodogram case $(J=7)$

British Pound, Canadian Dollar, German Mark, Swiss Franc and Japanese Yen. In particular, in order to implement our multifractal analysis, we assume that the dynamic Hurst exponent $H(t)$ is a stepwise constant function whose intervals are determined by splitting up each time series into four non-overlapping sub-periods: June 1972 to July 1976; August 1976 to January 1982; February 1982 to June 1987; and July 1987 to September 1994. The choice of these four time sub-periods is driven by the (relative) homogeneity of the economic and political conditions in each geographical region.

In table $1 \mathrm{~A}$ to table $1 \mathrm{~F}$, we report some standard descriptive statistics. The quantities reported indicate the number of observations, the minimum and maximum values of the time series, the means, the medians, the standard deviations, the skewness, and the kurtosis.

Generally, all the considered time series qualitatively denote to some degree a departure from normality. This is evidenced by the medians that differ from the corresponding means, skewness values, and particularly, kurtosis values. These departures are also confirmed by the performance of a simple $\chi^{2}$-type test for distribution fitting, which rejects the null hypothesis of normality for all the time series at $1 \%$ significance level.

From a short and medium-term autocorrelation point of view, we investigate the sample autocorrelation function up to lag 22 (about a onemonth trading period). In general, with the exception of certain time 


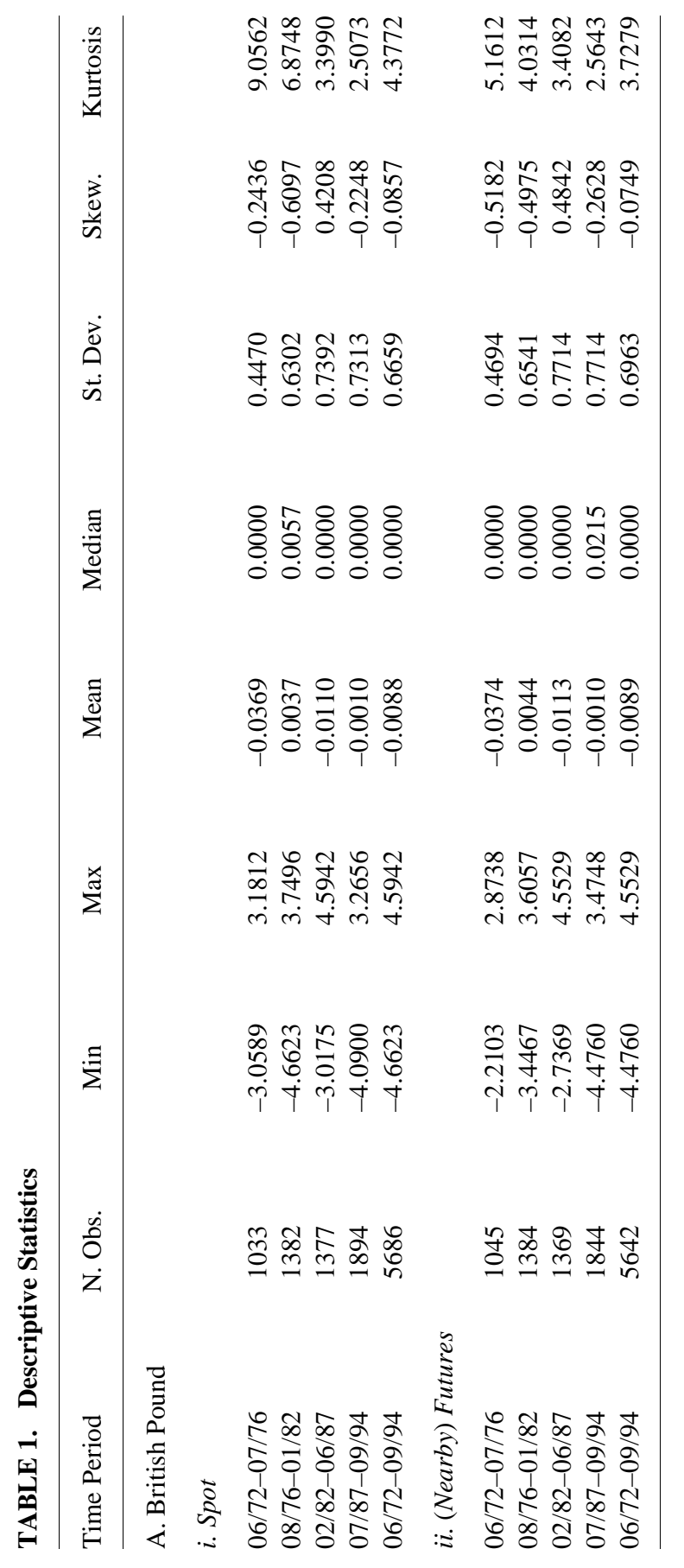




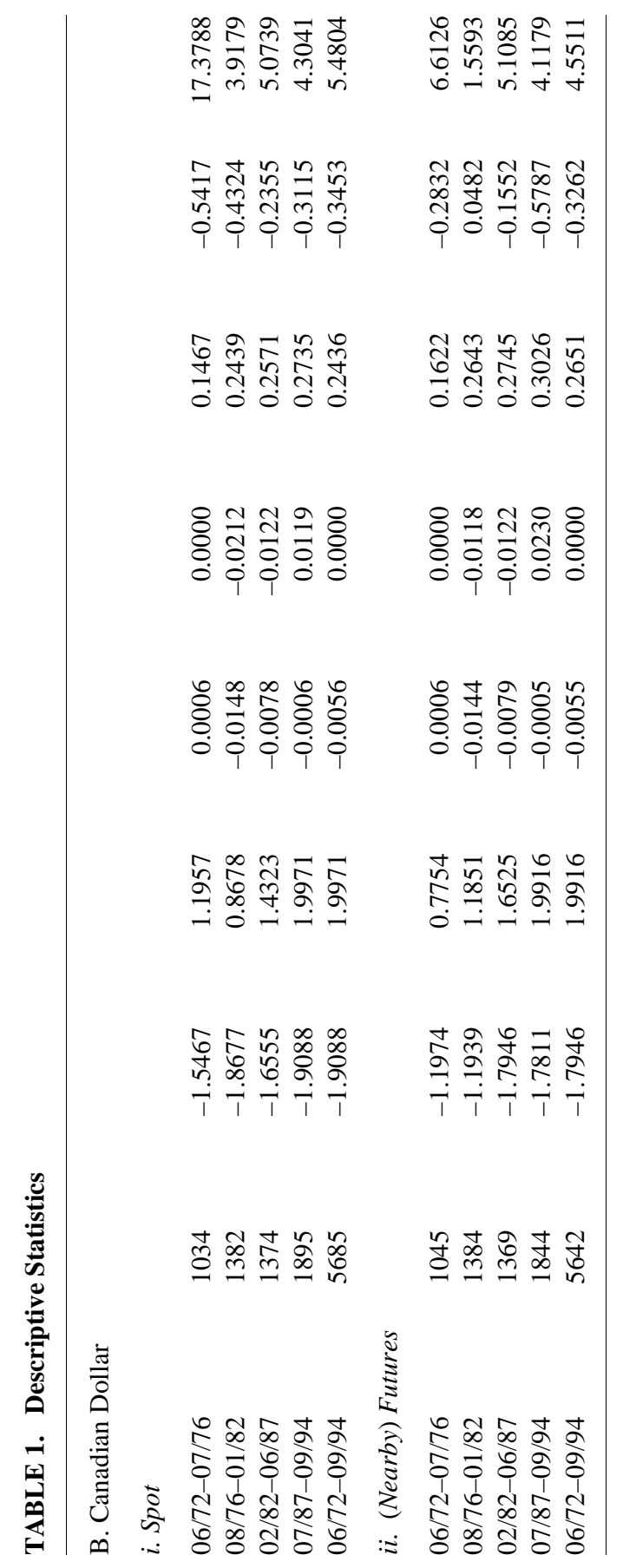




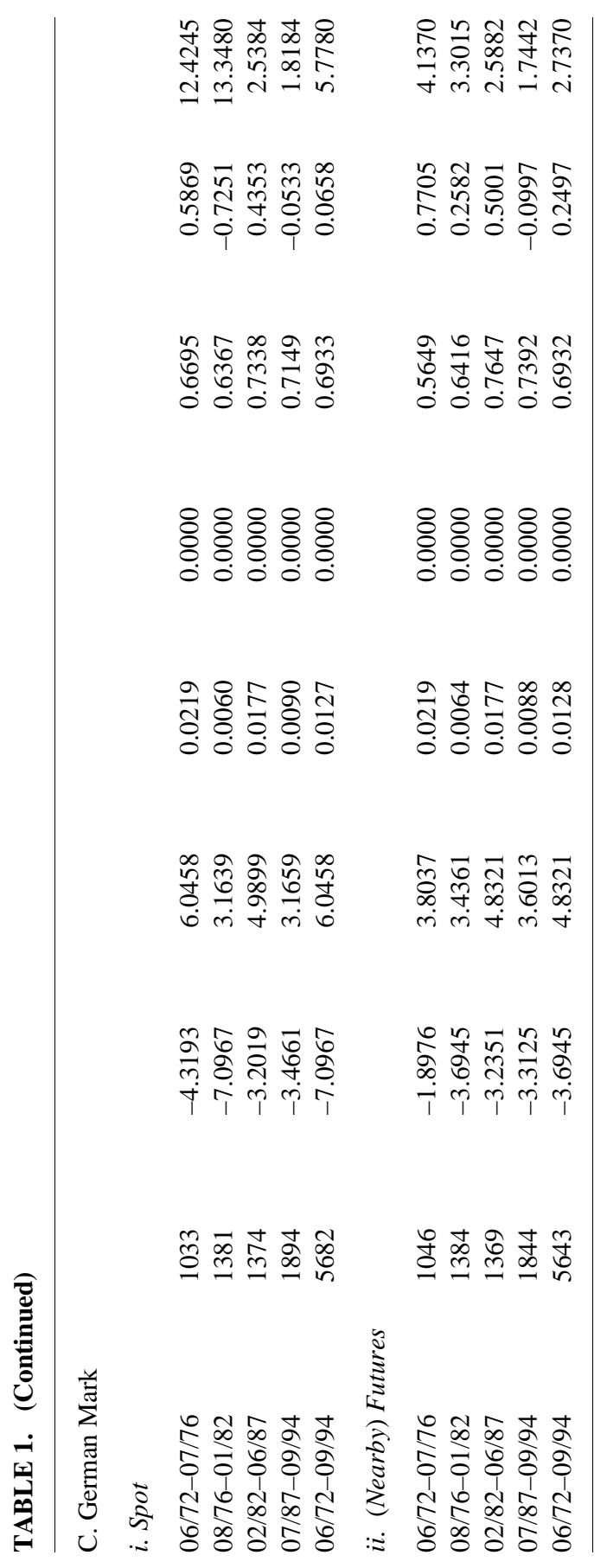




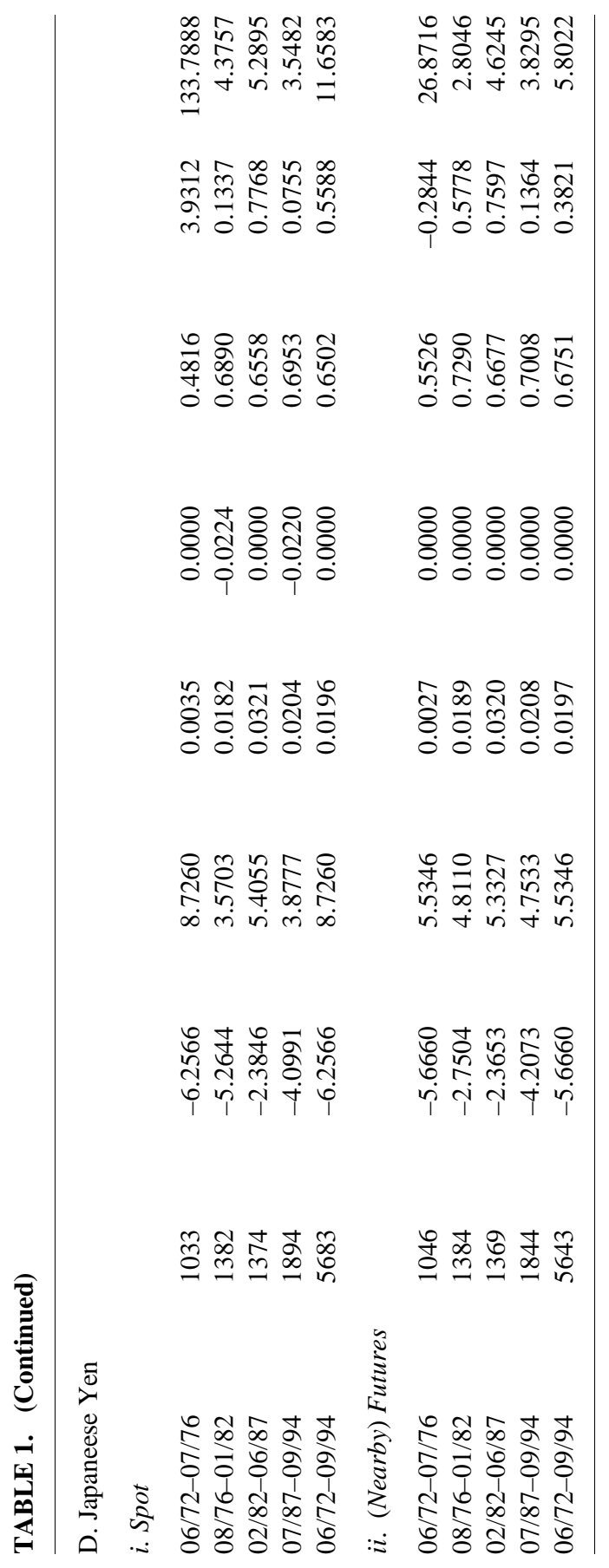




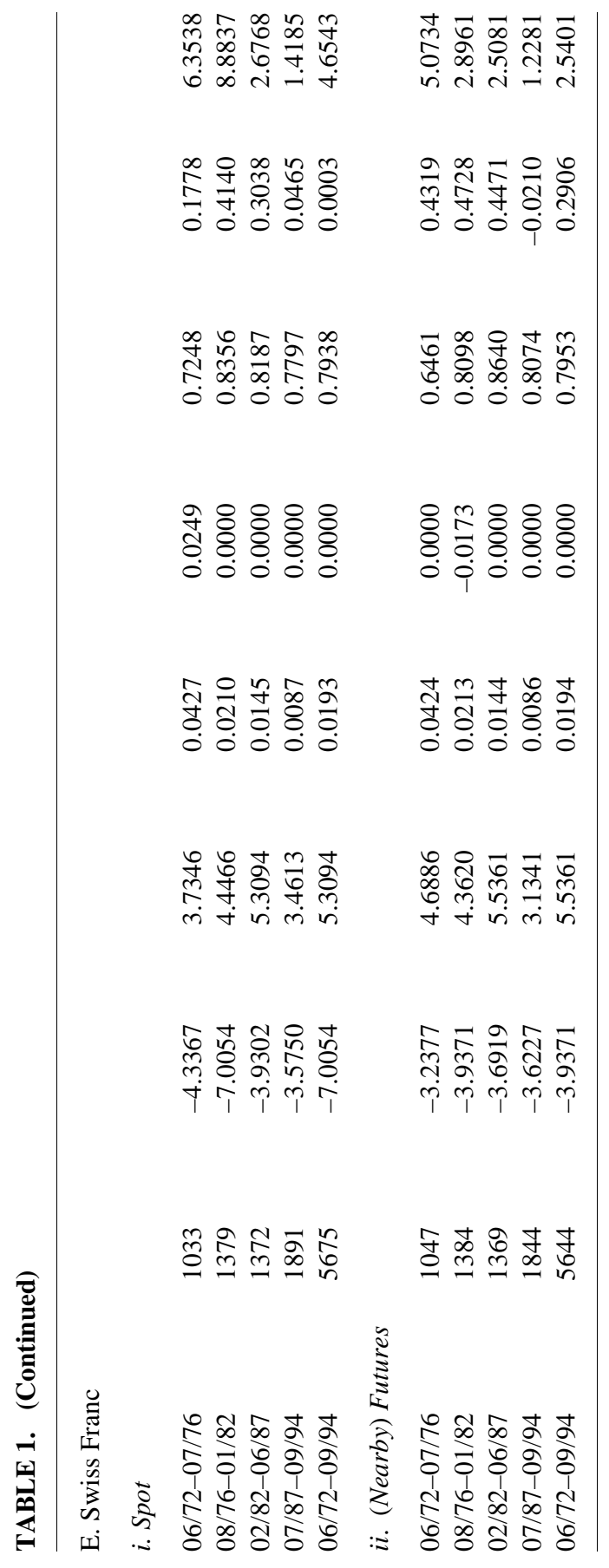




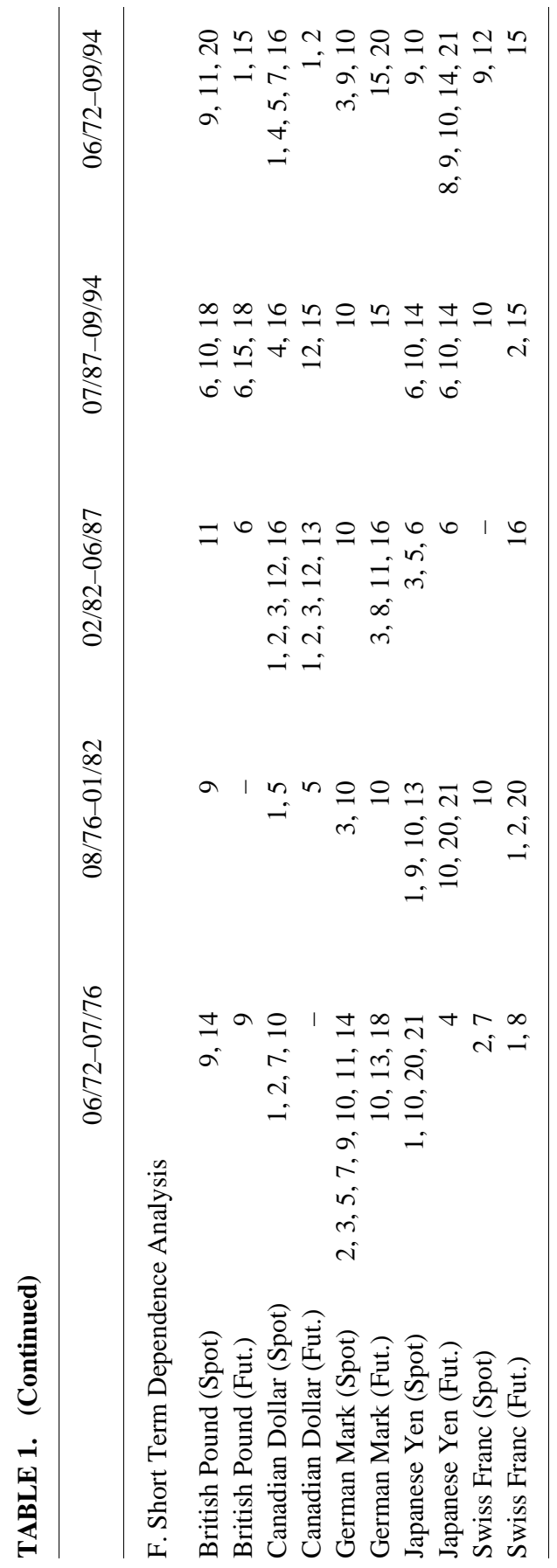


series, such an autocorrelation structure is negligible. In table $1 \mathrm{~F}$, we report the lag(s) for which the corresponding autocorrelation coefficient is significantly different from 0 at the $5 \%$ significance level for each time series under observation. ${ }^{17}$

Finally, some authors, such as Lobato and Savin (1998), suggest that evidence of long-term memory could be spuriously caused by nonstationarity in the time series itself. To test for non-stationarity, we perform the basic Dickey-Fuller test and its properly augmented version. ${ }^{18}$ For all the considered series, both tests reject the null hypothesis of non-stationarity (more precisely the tests reject the presence of a unit root in the autoregressive representation) at the $2 \%$ significance level. ${ }^{19}$

\section{Empirical Results of MultiFractal Analysis ${ }^{20}$}

The empirical results obtained are reported in table $2 \mathrm{~A}$ to table $2 \mathrm{E}$. In particular, the results relative to each of the considered single time periods are presented in four rows. The first three rows are devoted to the modified $R / S$-based approach, and the fourth row is devoted to the periodogram-based approach. In the columns labeled "*” we report the information concerning the assumed short-term dependence structure (in the first three rows relative to each period), and the bandwidth value (in the fourth row relative to each period). In the columns labeled " $H_{0}$ " we report the results of the test for no long-term dependence (acceptance or non-rejection is indicated by "A", rejection is indicated by its significance level), and in the columns labeled " $H$ " we report the values of the Hurst exponent. ${ }^{21}$

17. Such as the the Canadian Dollar spot, the Canadian Dollar futures, the German Mark spot, the Japanese Yen spot, and the Swiss Franc futures in some sub and full-sample periods.

18. For more details see Dickey and Fuller $(1979,1981)$.

19. The $2 \%$ significance level is the lowest boundary of the significance levels tabulated in Dickey and Fuller (1979).

20. Statistical computations were performed by Marco Corazza.

21. Notice that, although for completeness of exposition we also report the cases when the null hypothesis is rejected at the $20 \%$ significance level, practically we consider such rejections as acceptances in table $2 \mathrm{~F}$. 


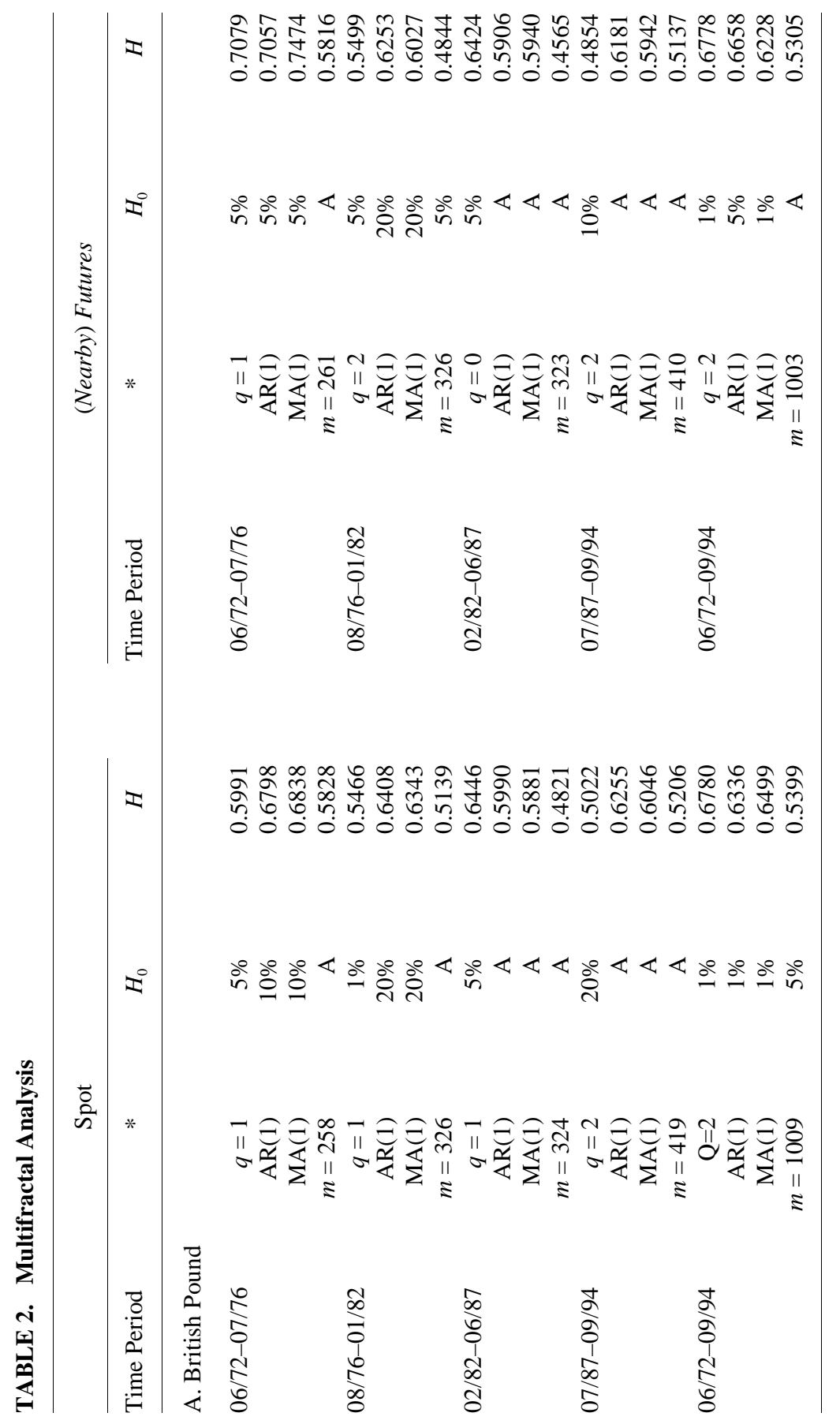




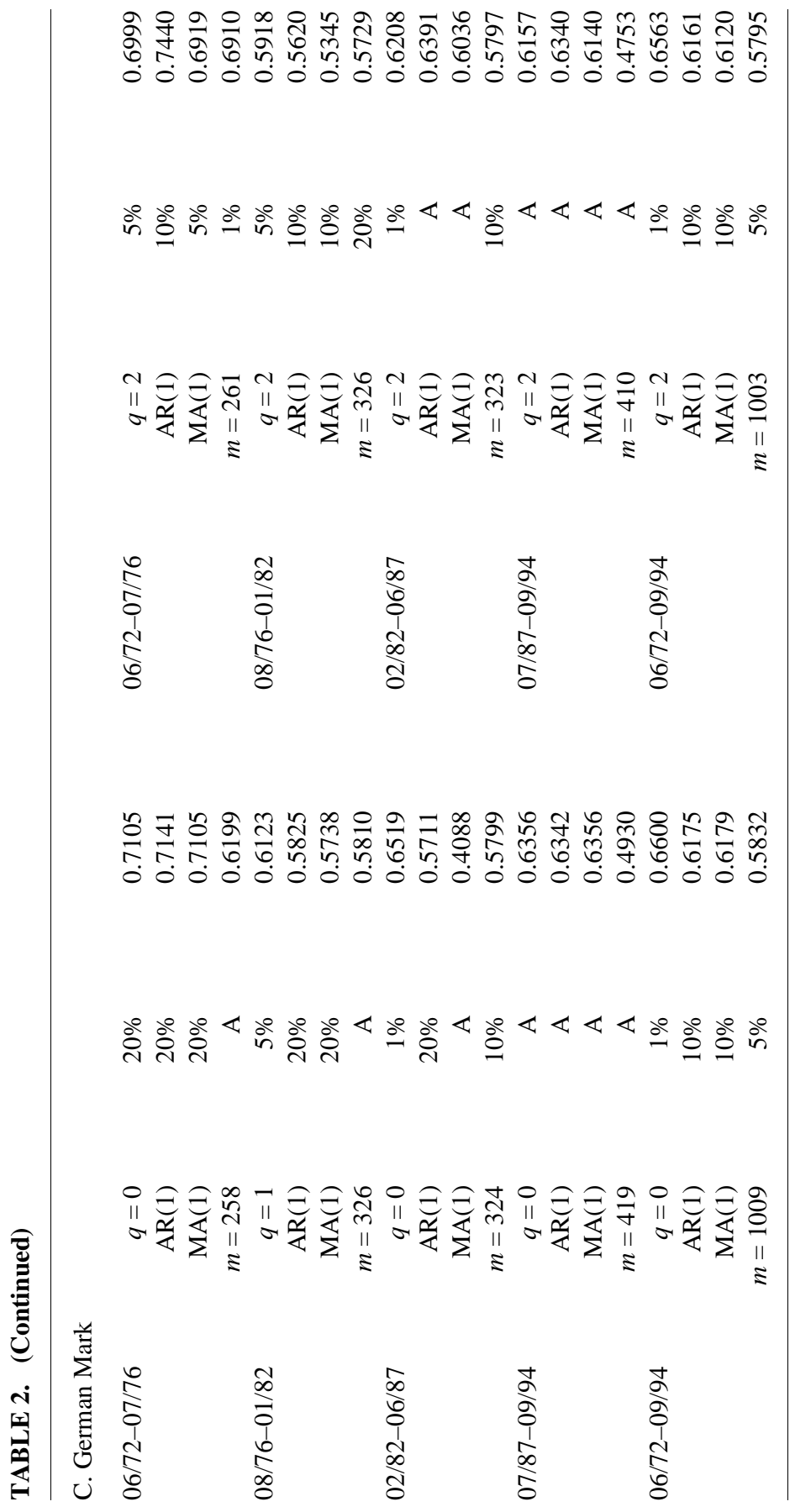




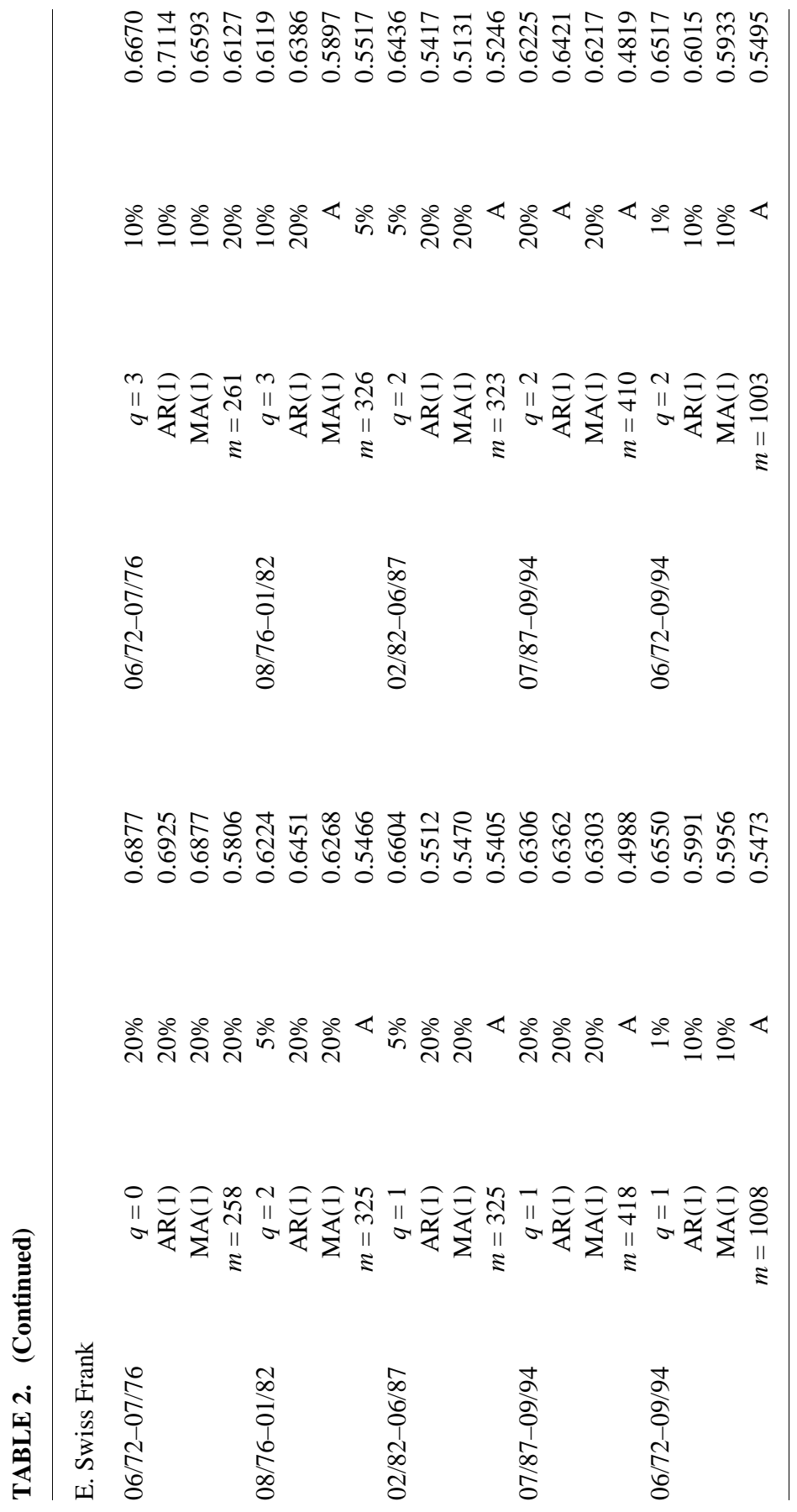




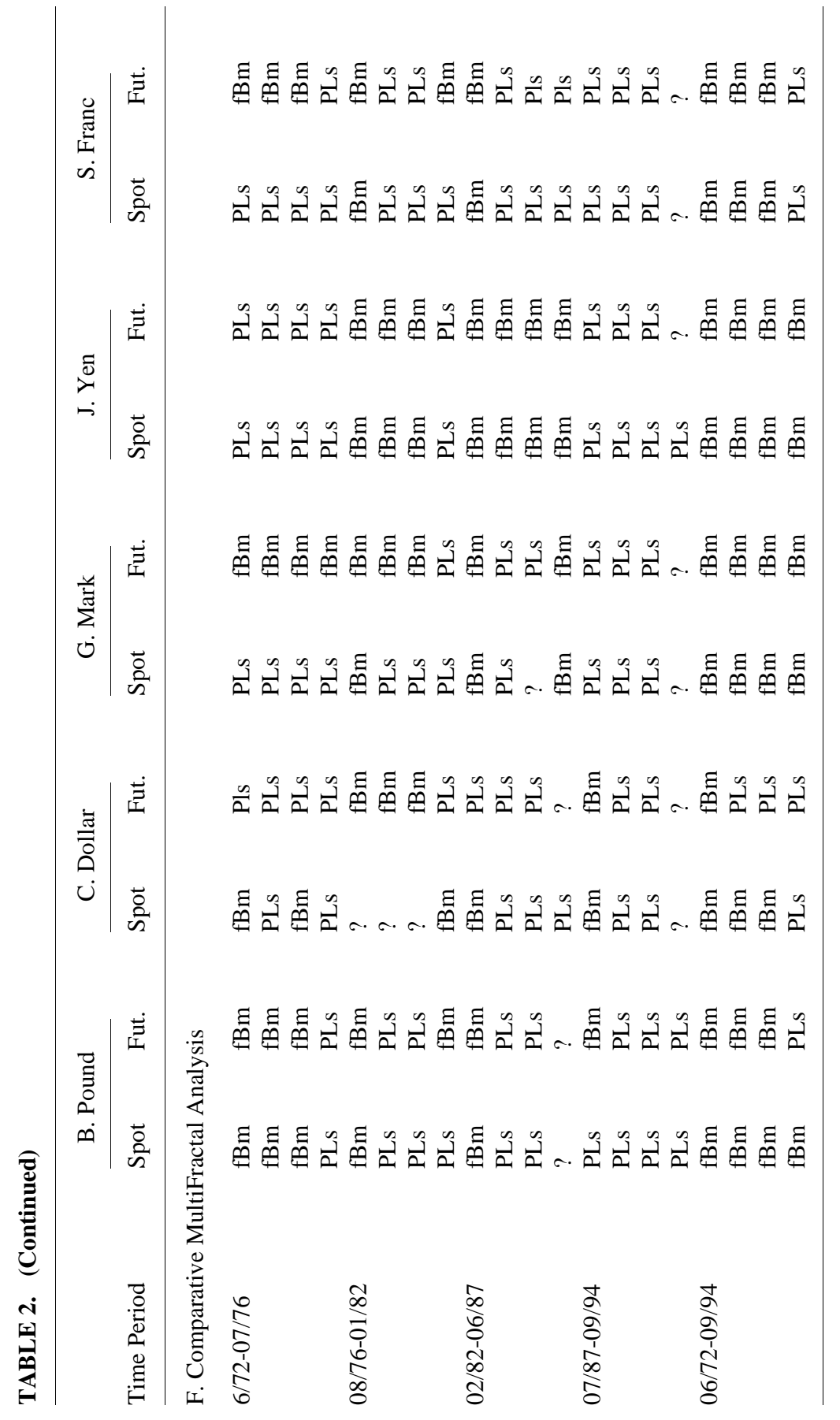


Generally, from the results reported in Table 2A to Table 2E, we observe that for the $66 \%$ of the considered time periods, both the modified $R / S$-based and the periodogram-based-tests qualitatively agree to accept or reject the null hypothesis of no long-term memory. ${ }^{22}$

We also wish to note that, in general, the estimates of $H$ based on the modified $R / S$ approach are greater than the corresponding estimates based on the periodogram approach. This is in accordance with the findings of Mandelbrot and Wallis (1969) and Jacobsen (1996), which confirm that the modified $R / S$-based estimation procedure overestimates the value of $H$ when the true value is lower than 0.72 (as it seems to be in the majority of our cases).

Again, for all time periods and for both spot and (nearby) futures foreign currency markets, the corresponding value of the dynamic Hurst exponent $H(t)$ is neither equal to 0.5 nor constant over time. This provides us with important empirical evidence for the MFMH or, at least, for the need to revise the EMH. In particular, the dynamic dimension is well supported by the test for no long-term dependence results. In fact, both the spot and (nearby) futures foreign currency markets are characterized over time by different underlying stochastic processes: the $\mathrm{fBm}$, the PLs motion and an undetectable one. ${ }^{23}$

Almost all the fBms describing the stochastic behavior of a wide percentage of the time sub-periods show a persistent long-term dependence, that is $H \in(0.5,1)$, and all the PLs motions describing the stochastic behavior of another wide percentage of the time sub-periods are distinguished by the non-finiteness of the variance, that is by $\alpha \in(1$, 2) (by $\alpha=1 / H$ ). Coupling both these aspects, that is, long-term dependence/independence and variance finiteness/non-finiteness, it follows that the structure of financial risk can vary widely from one time sub-period to the next.

In general, the spot and the (nearby) futures foreign currency

22. There are instances when at least two of the three sub-cases of the modified R/Sbased approach $(q=\#, \operatorname{AR}(1)$, and MA(1)) qualitatively agree with the only accept/reject decision given by the periodogram-based approach.

23. For these processes jointly characterized by $H \in(0,0.5)$ and long-term independence, some authors, such as Evertsz (1995a, 1995b), suggest suitable mixtures of fBms and PLs motions. Others, like Zou (1996) suggest that some proper PLs distribution sub-families, such as a fractional distribution may be suitable. These issues have not been settled and are beyond the scope of this work. 
markets for each currency are characterized by similar dynamic stochastic structures, especially from a short and long-term dependence/independence point of view.

\section{Economic Interpretations}

In general, all the analyzed foreign currency markets exhibit a behavior over time influenced by their Hurst exponent and by their long-term independence/dependence. This behavior provides empirical support for the MFMH as a reasonable extension of the EMH. In fact, different stochastic processes describe the foreign currency markets during various periods. We distinguish three phases characterizing the conjectured MFMH (instead of the two standard ones): a "regular" phase, a new phase that we identify as "semi-regular" and an "irregular" phase.

The "regular" phase is associated with the $\mathrm{fBm}$ via long-term dependence, that is, with the Hurst exponent $H \in(0.5,1)$. In fact, the characteristics of the financial risk described by the corresponding distributional law are such as to permit a relatively simple matching between the demand and supply for two reasons:

First, the statistical self-similarity characterizing the fBms guarantees that the risk associated with investments of different horizon lengths $t$ and $a t$, with $a>0$, are evaluated in the same proportion by their corresponding investors. Actually, $\left\{B_{H}(t), t \geq 0\right\}$ and $\left\{a^{-H} B_{H}(a t), \mathrm{t} \geq 0\right\}$, with $a>0$, have the same distributional law. ${ }^{24}$ Because of this, the demands and supplies of these investors with different horizon lengths match, and thus ensure a certain liquidity for the foreign currency markets. Notice that the statistical self-similarity implicitly asserts the existence of some relationships between the Hurst exponent, $H$, and the liquidity level.

Second, the long-term persistent memory distinguishing these foreign currency markets makes it possible to partially forecast future returns, and consequently, ex ceteris paribus, to manage a lower risk than in the classical independently and identically log-normally distributed

24. Notice that $a^{-H}$ plays the role of a proportionality factor. 
environment. ${ }^{25}$ This lowering of long-run risk may explain the attractiveness of longer investment horizons by some investors in an $\mathrm{fBm}$ regime.

In particular, in order to explain such long-term persistent memory, we can conjecture that the analyzed foreign currency markets are characterized by the regular arrival of new information confirming underlying economic trends. Of course, this reduces the spread between the ability of economic agents to make optimal decisions and the complexity of decisions made under uncertainty.

The "semi-regular" phase is associated with the PLs motion, that is distinguished both by the non-finiteness of the variance because of $\alpha \in$ $(1,2)$, and by the no long-term dependence. The characteristics of the financial risk arising from the corresponding distributional law permit, again, the matching between the demand and the supply, but to a lower degree as compared to the "regular" phase. In fact, in the current case, the only source of attractiveness for investors who value lengthy horizons is the statistical self-similarity. In particular, notice that the values of the Hurst exponents, characterizing the "regular" and the "semi-regular" phases are within a limited range and, so, their impacts on the liquidity levels are quite similar for both phases. At least, no significant differences are apparent. To the contrary, the unpredictability of future returns due to the absence of some long-term dependence puts the "semi-regular" phase volatility in a higher risk class than does the unpredictability of the "regular" phase (however, ex ceteris paribus, both normal). Furthermore, the distributional properties of the underlying stochastic process put this PLs volatility in a higher risk class than the normal one ${ }^{26}$ Of course, this latter financial risk characteristic causes a lower participation of investors in the "semi-regular" foreign currency market than in the "regular" foreign currency market and, in particular, a lower participation of investors having long horizon lengths that are associated with highest risk. Because of this, in the corresponding "semiregular" foreign currency market there are both a lower liquidity level, and a lower average investment horizon length than in the "regular" phase foreign currency one.

25. Notice that, because of the trend due to long-term dependence, the standard deviation of the considered fBms provides an over-evaluation of the actual volatility of the corresponding foreign currency markets.

26. Recall that the tails of the PLs motions with $\alpha \in(0,2)$ decay slower than the fBm ones. 
In order to explain such a higher risk level distinguishing the "semiregular" phase, we can conjecture that the corresponding foreign currency markets are characterized by an irregular arrival of exogenous noise. Of course, this makes it difficult for investors to detect any trends that may exist in the fundamentals of the economy and thus may influence their ability to make rational decisions.

The "irregular" phase is associated with an undetectable stochastic process, that may be a suitable mixture of fBms and PLs motions, or which may belong to some proper PLs distribution sub-family. Although such lack of detection is possible, the (generic) identifiable characteristics of the corresponding distributional law (and, consequently, of the financial risk) are such as to prevent a simple matching between the demand and supply. In fact, in this "irregular" phase, volatility belongs to a risk class quite similar to the one that characterizes the "semi-regular" phase. Again, this causes primarily a lower participation of investors having long horizon lengths (who are associated with a higher level of risk) and, consequently, a lower liquidity level and a lower average investment horizon length than in the "regular" phase foreign currency markets. Moreover, the underlying stochastic process may or may not be characterized by the statistical self-similarity. In the first case, for the "irregular" phase, the corresponding Hurst exponent, $H$, is lower than that for the "regular" and "semi-regular" phases. It is simple to prove, under a reasonable assumption on $a$, that the proportionality factor $a^{-H}$ is higher for these latter phases. ${ }^{27}$ In the second case different horizon length investors do not evaluate investments in the same proportional way, and so their demands and supplies do not match.

In particular, in order to explain such a financial environment, we can conjecture that the corresponding foreign currency markets are characterized by the arrival of conflicting information. This causes very different and, often, incompatible behavior among the economic agents.

\section{Concluding Remarks}

All the foreign currency markets studied in this article exhibit a Hurst exponent that is statistically different from 0.5 in the majority of the samples studied. Furthermore, it is also found that the Hurst exponent is 
not fixed but it changes dynamically over time. The interpretation of these results is that the foreign currency returns follow either a fractional Brownian motion or a Pareto-Levy stable distribution. The key question is: what are the implications of such findings on the Efficient Market Hypothesis? Both in its original formulation and in the recent more sophisticated elaborations of the random walk hypothesis found in Campbell, Lo and MacKinlay (1997), the efficient market hypothesis is associated with returns that follow a Brownian motion with Hurst exponent equal to 0.5. Rogers (1997) has shown that a market where the asset returns follow a fractional Brownian motion cannot be efficient since there always exists an arbitrage strategy. Our approach has been to use the statistical evidence in this article to support the proposed Multi-Fractal Market Hypothesis. Needless to say, this extension of the traditional Efficient Market Hypothesis needs a further elaboration that goes beyond the general ideas we have offered in the previous sections. In particular, we need to develop theoretical explanations for both longterm positive and negative dependence as well as explanations for the transition of distributions from Brownian to fractally Brownian or Pareto-Levy stable.

\section{References}

Alexakis, P., and Apergis, N. 1996. ARCH effects and cointegration: Is the foreign exchange market efficient? Journal of Banking and Finance 20 (4): 687-697.

Andrews, D.W.K. 1991. Heteroscedasticity and autocorrelation consistent covariance matrix estimation. Econometrica 59 (5): 817-858.

Belkacem, L.; Levy Vehel, J.; and Walter, C. 1996. CAPM, risk and portfolio selection in stable markets. Rapport de Recherche n. 2776, INRIA, Le Chesnay Cedex.

Bleaney, M., and Mizen, P. 1996. Nonlinearities in exchange rate dynamics: Evidence from five currencies. 1973-1994. Economic Record 72(216): 36-45.

Campbell, J.Y.; Lo, A.W.; and MacKinlay, A.C. 1997. The econometrics of financial markets. Princeton University Press, Princeton.

Chan, K.; Gup, B.; and Pan, M.S. 1992. Market efficiency and cointegration tests for foreign currency futures markets. Journal of International Financial Markets, Institutions \& Money 2(1): 78-89.

Cheung, Y.-W., and Lai, K.S. 1993. Do gold market returns have long memory? The Financial Review 28(2): 181-202. 
Chiang, T.C., and Jiang, C.X. 1995. Foreign exchange returns over short and long horizons. International Review of Economics and Finance 4(3): 267282.

Corazza, M. 1996. Long-term memory stability in the Italian stock market. Economics \& Complexity 1(1): 19-28.

Corazza, M.; Malliaris, A.G.; and Nardelli, C. 1997. Searching for fractal structure in agricultural futures Markets. The Journal of Futures Markets 17(4): 433-473.

Cornell, B. 1977. Spot rates, forward rates and exchange market efficiency. Journal of Financial Economics 5: 55-65.

Delgado, M.A., and Robinson, P.M. 1996. Optimal spectral bandwidth for long memory. Statistica Sinica 6(1): 97-112.

Dickey, D.A., and Fuller, W.A. 1979. Distribution of the estimators for autoregressive time series with a unit root. Journal of the American Statistical Association 74: 427-431.

Dickey, D.A., and Fuller, W.A. 1981. Likelihood ratio statistics for autoregressive time series with a unit root. Econometrica 49: 1057-1072.

Evertsz, C.J.G. 1995a. Fractal geometry of financial time series. Fractals 3(3): 609-616.

Evertsz, C.J.G. 1995b. Self-Similarity of high-frequency USD-DEM exchange rates. Proceedings of the First International Conference on High Frequency Data in Finance, Zurich.

Evertsz, C.J.G., and Berkner, K. 1995. Large deviation and self-similarity analysis of graphs: DAX stock prices. Chaos, Solitons \& Fractals 6: 121-130.

Falconer, K. 1990. Fractal geometry. John Wiley \& Sons, New York.

Fama, E.F. 1970. Efficient capital markets: Review of theory and empirical work. The Journal of Finance 25: 383-417.

Fama, E.F. 1991. Efficient capital markets: II. The Journal of Finance 46(5): 15751617.

Fang, H.; Lai, K.S.; and Lai, M. 1994. Fractal structure in currency futures price dynamics. The Journal of Futures Markets 14(2): 169-181.

Frankel, J.A. 1980. Tests of rational expectations in the forward exchange market. Southern Economic Journal 46: 1083-1101.

Hsieh, D.A. 1989. Testing for nonlinear dependence in daily foreign exchange rates. Journal of Business 62(3): 339-368.

Hsieh, D.A. 1992. A Nonlinear stochastic rational expectations model of exchange rates. Journal of International Money and Finance 11(3): 235250 .

Jacobsen, B. 1996. Long term dependence in stock returns. Journal of Empirical Finance 3: 393-417.

Kao, G.W., and Ma, C. 1992. Memories, heteroskedasticity, and price limit in currency futures markets. Journal of Futures Markets 12(6): 679-692.

Kho, B.C. 1996. Time varying risk premia, volatility, and technical trading rule 
profits: Evidence from foreign currency futures markets. The Journal of Financial Economics 41(2): 249-290.

Leachman, L.L., and El Shazly, M. R. 1992. Cointegration analysis, error correction models and foreign exchange market efficiency. Journal of International Financial Markets, Institutions \& Money 2(1): 57-77.

Levich, R.M., and Thomas, L.R. 1993. The Significance of technical trading rule profits in the foreign exchange markets: A bootstrap approach. Journal of International Money and Finance 12(5):451-474.

Lévy, P. 1925. Calcul des probabilites. Gauthier-Villars, Paris.

Liu, Y. A.; Pan, M.S.; and Hsueh, L.P. 1993. A modified R/S Analysis of longterm dependence in currency futures prices. Journal of International Financial Markets, Institutions \& Money 3(2): 97-113.

Lo, A.W. 1991. Long-term memory in stock market prices. Econometrica 59(5): 1279-1313.

Lo, A.W., and MacKinlay, A.C. 1988. Stock Market Prices Do not follow random walks: Evidence from a simple specification test. Review of Financial Studies 1: 41-66.

Lobato, I., and Robinson, P.M. 1996. Averaged periodogram estimation of long memory. Journal of Econometrics 73: 303-324.

Lobato, I.N., and Savin, N.E. 1998. Real and spurious long memory properties of stock market data. preprint, University of Iowa, Iowa City.

Machones, M.; Mase, S.; Plunkett, S.; and Thrash, R. 1994. Price prediction using nonlinear techniques. The Magazine of Artificial Intelligence in Finance Fall: 51-56.

Mandelbrot, B.B., and Van Ness J.W. 1968. Fractional Brownian motions, fractional noises and applications. SIAM Review 10(4): 422-437.

Mandelbrot, B.B., and Wallis, J.R. 1969. Robustness of the rescaled range R/S in the measurement of noncyclic long run statistical dependence. Water Resources Research 5: 967-988.

Mittnik, S., and Rachev, S.T. 1993. Reply to comments on modeling asset returns with alternative stable distributions and some extensions. Economic Review 12: 347-390.

Ostasiewicz, W. 1996. Advanced financial techniques. Badania Operacyine $i$ Decyzje 3-4: 161-178.

Pancham, S. 1994. Evidence of the multifractal market hypothesis using wavelet transforms. mimeo, Florida International University, Miami (downloadable from the web site: http://home.nyc.rr.com/spancham).

Peltier, R.F., and Levy Vehel, J. 1994. A new method for estimating the parameter of fractional Brownian motion Rapport de Recherche n. 2396, INRIA, Le Chesnay Cedex.

Peltier, R.F., and Levy Vehel, J. 1995. MultiFractional Brownian motion: Definition and preliminary results. Rapport de Recherche n. 2645, INRIA, Le Chesnay Cedex. 
Peters, E.E. 1991. Chaos and order in the capital markets. John Wiley \& Sons, New York.

Peters, E.E. 1994. Fractal market analysis. John Wiley \& Sons, New York.

Robinson, P.M. 1994. Semiparametric analysis of long-memory time series. Annals of Statistics 22: 515-539.

Robinson, P.M. 1994a. Rates of convergence and optimal spectral bandwidth for long range dependence. Probability Theory and Related Fields 99: 443-473.

Robinson, P.M. 1994b. Time series with strong dependence, in sims. C.A. (ed.) Advances in Econometrics. Sixth World Congress 1, Cambridge University Press: 47-95.

Rogers, L.C.G. 1997. Arbitrage with fractional Brownian motion. Mathematical Finance 7: 95-105.

Samuelson, P.A. 1965. Proof that properly anticipated prices fluctuate randomly. Industrial Management Review 6: 41-49.

Shubik, M. 1997, Proceedings of a conference on risks involving derivatives and other new financial instruments. Economic Notes 26: 169-443.

Taqqu, M.S. 1986. A bibliographical guide to self-similar processes and longrange dependence. In Eberlein, E. and Taqqu, M.S. (eds.) Dependence in Probability and Statistics, Birkhauser, Boston: 137-162.

Taqqu, M.S.; Teverovsky, V.; and Willinger, W. 1995. Estimators for long-range dependence: An empirical study. Fractals 3(4): 785-798.

Taylor, S.J. 1992. Rewards available to currency futures speculators: Compensation for risk or evidence of inefficient pricing? Economic Record, Supplement: 105-116.

Van De Gucht, L.M.; Dekimpe, M.G.; and Kwok, C.Y. 1996. Persistence in foreign exchange rates. Journal of International Money and Finance 15(2): 191-220.

Zhou, B. 1996. High frequency data and volatility in foreign-exchange rates. Journal of Business and Economic Statistics 14(1): 44-52. 